\title{
Recognizing Rotated Faces from Two Orthogonal Views in Mugshot Databases
}

\author{
Xiaozheng Zhang, Yongsheng Gao \\ Computer Vision \& Image Processing Lab, \\ School of Engineering, Griffith University, \\ Australia \\ $\{x . z h a n g, y o n g s h e n g . g a o\} @$ griffith.edu.au
}

\author{
Bai-ling Zhang \\ School of Computer Science and Mathematics, \\ Victoria University, \\ Australia \\ bzhang@csm.vu.edu.au
}

\begin{abstract}
Tolerance to pose variations is one of the key remaining problems in face recognition. It is of great interest in airport surveillance systems using mugshot databases to screen travellers' faces. This paper presents a novel poseinvariant face recognition approach using two orthogonal face images from mugshot databases. Virtual views under different poses are generated in two steps: shape modeling and texture synthesis. In the shape modeling step, a feature-based multilevel quadratic variation minimization approach is applied to generate smooth $3 D$ face shapes. In the texture synthesis step, a non-Lambertian reflectance model is explored to synthesize facial textures taking into account both diffuse and specular reflections. A view-based face recognizer is used to examine the feasibility and effectiveness of the proposed pose-invariant face recognition. The experimental results show that the proposed method provides a new solution to the problem of recognizing rotated faces.
\end{abstract}

\section{Introduction}

Computerized human face recognition is an active research area in the field of computer vision and pattern recognition. Appearance changes due to illumination condition, facial expression, and face rotations are three main issues that affect the performance of a face recognition system. Compared with illumination and expression changes, recognizing rotated faces is more challenging and remains unsolved [1], though it is of great interest in many face recognition applications such as surveillance systems.

Prior work shows that it is realistic yet challenging to perform view-based pose invariant face recognition using synthesized virtual views. 2D techniques and 3D models were used to predict the appearance of a human face under different poses. Beymer and Poggio [2] mapped 2D facial transformations observed on a prototype face onto a novel, non-prototype face to generate virtual views for recognition. Cootes et al. [4] proposed a 2D statistical method to approximate facial appearances from novel viewpoints. However, 2D techniques cannot cover large rotations, since they limit the appearance estimation to pose transformations [13]. Georghiades et al. [6] introduced a generative 3D illumination cone model trained from seven gallery images for virtual view synthesis. Morphable 3D face models [3, 9] were proposed using prior knowledge of human head geometries and they adjusted shape and texture coefficients according to individual faces. For face texture analysis, the Lambertian reflectance model is currenctly popular [2, 6, 8]. However, human face surfaces reflect incident lights both diffusely and specularly and therefore cannot be approximated as Lambertian surfaces [5]. Ip and Yin [9] analyzed the virtual texture of an arbitrary view by interpolating the frontal texture and the profile texture. Blanz and Vetter [3] included Phong reflectance model and calculated facial textures from a single real view using an unstable nonlinear optimization method. A stable texture analysis approach taking into account both diffuse and specular reflections is desirable for virtual view synthesis.

This paper attempts to address the problem of recognizing rotated faces when only frontal and profile face views per person are available. For example, an airport surveillance system needs to recognize travellers' faces under arbitrary poses based on mug shot databases. A novel featurebased 3D face modeling approach is proposed to generate personalized 3D face shapes. Then a stable texture analysis approach is presented considering both diffuse and specular reflections (non-Lambertian). Combined with an Eigenface recognizer, the virtual views are used for pose-invariant face recognition.

\section{3D face shape modeling}

In this paper, a two-step approach consisting of shape modeling and texture analysis is proposed to generate virtual face views from two orthogonal gallery views (shown 


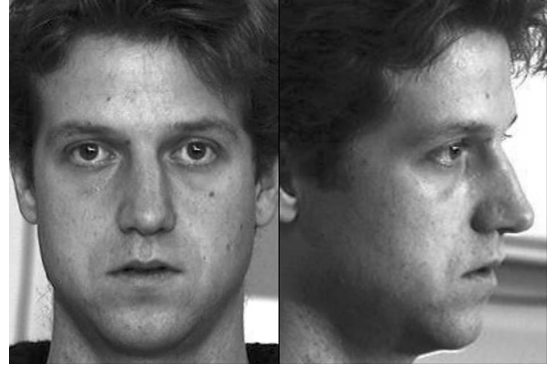

Figure 1. The two orthogonal face views used as gallery images.

in Fig. 1) for pose-invariant face recognition. In the step of face shape modeling, a feature-based multilevel quadratic variation minimization approach [12] is applied to reconstruct 3D face shapes. This method utilizes a hierarchical coarse-to-fine strategy to generate a 3D face surface from a number of constraint points. Suppose a 3D face shape is formulated as a depth graph $s=s(x, y)$ where $(x, y)$ corresponds to a pixel in a frontal face view. Given a set of constraint points $\delta$ in the frontal image, the depth of constraint point $(x, y) \in \delta$ is the horizontal position $c(x, y)$ of point $(x, y)$ in the profile view. A coarse-to-fine hierarchy of control lattices, $\Phi_{0}, \Phi_{1}, \ldots, \Phi_{w}$, is introduced to generate a sequence of surface reconstructions at different resolution levels. In this paper, the starting coarsest control lattice is chosen as $\Phi_{0}=4 \times 4$, and the finest control lattice is $\Phi_{7}=512 \times 512$. We denote the spacing in $\Phi_{0}$ as $h_{0}=128$. The spacings in $\Phi_{k}$ and $\Phi_{7}$ are $h_{k}=h_{k-1} / 2$ and $h_{7}=1$, respectively.

At control lattice $\Phi_{k}$ where $h_{k} \times h_{k}$ pixels merge into a single grid, pixel $(x, y)$ is mapped to grid $(u, v)$ where $u=$ $\left\lfloor x / h_{k}\right\rfloor$ and $v=\left\lfloor y / h_{k}\right\rfloor$. The constraint point set becomes $\delta_{k}=\left\{\left(\left\lfloor x / h_{k}\right\rfloor,\left\lfloor y / h_{k}\right\rfloor\right) \mid(x, y) \in \delta\right\}$. If a new constraint point $(u, v) \in \delta_{k}$ includes two or more original constraint points, the depth $c_{k}(u, v)$ is the average depth of these original constraint points, otherwise $c_{k}(u, v)=c(x, y)$ where $u=\left\lfloor x / h_{k}\right\rfloor$ and $v=\left\lfloor y / h_{k}\right\rfloor$. Under $\Phi_{k}$, a quadratic variation minimization [7] is performed which generates a $3 \mathrm{D}$ surface by minimizing a cost function

$$
\Theta\left(s_{k}\right)=\iint\left\{\left(\frac{\partial^{2} s_{k}}{\partial u^{2}}\right)^{2}+2\left(\frac{\partial^{2} s_{k}}{\partial u \partial v}\right)^{2}+\left(\frac{\partial^{2} s_{k}}{\partial v^{2}}\right)^{2}\right\} d u d v,
$$

subject to $s_{k}(u, v)=c_{k}(u, v), \forall(u, v) \in \delta_{k}$. In the minimization process, the neighbors of a constraint point move gradually towards the constraint point along $z$-axis (depth) while maintaining the smoothness of the surface represented by the objective function $\Theta\left(s_{k}\right)$. The result is smooth 3D surfaces around all the constraint points. The proposed hierarchical strategy enables points "far" away from all con- straint points to be neighbors of certain constraint points in a coarse level of control lattice and hence the minimization process can actually move them to better positions. By doing that, the approach is feasible to 3D surface modeling with "sparse" constraint points such as face modeling of high resolution and few facial features. The initial surface under $\Phi_{0}$ is

$$
s_{0}(u, v)= \begin{cases}0, & (u, v) \notin \delta_{0} \\ c_{0}(u, v), & (u, v) \in \delta_{0}\end{cases}
$$

The resulting surface $s_{k-1}^{*}$ under $\Phi_{k-1}$ is used as the initial surface under control lattice $\Phi_{k}$. The 3D face shape modeling approach is outlined in Algorithm 1.

\section{Algorithm 1. 3D face shape modeling}

\section{Iteration $(\delta, C)$ :}

comment: $\delta=$ original constraint point set,

$C=$ depths of constraint points;

(1) Initialization: $k \leftarrow 0, \Phi_{0}=4 \times 4, h_{0}=128$;

(2) $\delta_{0}=\left\{\left(\left\lfloor x / h_{0}\right\rfloor,\left\lfloor y / h_{0}\right\rfloor\right) \mid(x, y) \in \delta\right\}$, form $C_{0}$;

(3) Initialization: $s_{0}(u, v) \leftarrow\left\{\begin{array}{ll}0, & (u, v) \notin \delta_{0} \\ c_{0}(u, v), & (u, v) \in \delta_{0}\end{array}\right.$;

(4) $s_{0}^{*}=$ Quadratic VariationMinimization $\left(s_{0}, \delta_{0}, C_{0}\right)$;

for $k \leftarrow 1$ to 7

(5) $\Phi_{k}=2^{k+2} \times 2^{k+2}, h_{k}=2^{7-k}$;

(6) $\delta_{k}=\left\{\left(\left\lfloor x / h_{k}\right\rfloor,\left\lfloor y / h_{k}\right\rfloor\right) \mid(x, y) \in \delta\right\}$, form $C_{k}$;

(7) $s_{k}(u, v) \leftarrow\left\{\begin{array}{l}s_{k-1}^{*}(\lfloor u / 2\rfloor,\lfloor v / 2\rfloor),(u, v) \notin \delta_{k} \\ c_{k-1}(u, v), \quad(u, v) \in \delta_{k}\end{array} ;\right.$

(8) $s_{k}^{*}=$ QuadraticVariationMinimization $\left(s_{k}, \delta_{k}, C_{k}\right)$; (9) $s^{*}=s_{7}^{*}$;

comment: $s^{*}=$ resulting 3D surface model.

The multilevel quadratic variation minimization algorithm is applied to generate a smooth 3D face shape from frontal and profile face views as shown in Fig. 1. Facial features are manually specified on the two views to provide constraint points for the shape modeling. Fig. 2 indicates a sequence of face shape reconstructions under different control lattices generated from the two face views in Fig. 1. The face shapes are under $30^{\circ}$ (Fig. 1a,c) and $90^{\circ}$ (Fig. 1b) horizontal rotations in depth. The control lattices of the three rows are $16 \times 16$ (Fig. 2a), $64 \times 64$ (Fig. 2b), and $512 \times 512$ (Fig. $2 \mathrm{c}$, the finest). It takes $10^{4}$ iterations for each quadratic variation minimization and totally $8 \times 10^{4}$ iterations to converge.

\section{Facial texture synthesis}

In the second step, facial textures are analyzed from the pixel values of gallery views based on geometrical reflectance models. The most popular reflectance model in face texture analysis is Lambertian model, which assumes 


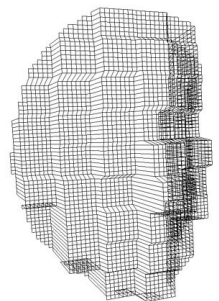

(a)

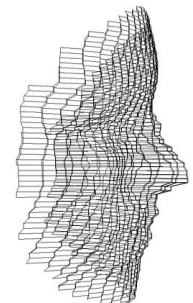

(b)

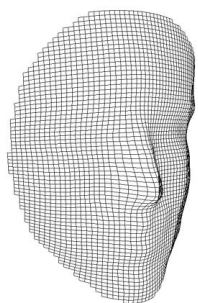

(c)

\section{Figure 2. The hierarchical face modeling pro-} cess.

that a face surface has only diffuse reflection independent of viewing conditions. However, Lambertian assumption does not hold since human faces exhibit both diffuse and specular reflections [5]. Phong reflectance model[10], which incorporates not only diffuse and ambient reflections, but also specular reflection, is expressed as

$$
I=k_{a} I_{a}+k_{d} I_{p o}(\vec{L} \cdot \vec{N})+k_{s} I_{p o}(\vec{R} \cdot \vec{V})^{n}
$$

where $I$ is the reflected intensity from a point on a face surface, which is also a pixel value on the face image, $k_{a}$ is the ambient albedo, $I_{a}$ is the ambient light intensity, $k_{d}$ is the diffuse albedo, $I_{p o}$ is the point light intensity, $\vec{L}$ is the direction of the point light, $\vec{N}$ is the surface norm, $k_{s}$ is the specular albedo, $\vec{V}$ is the viewing direction, and $\vec{R}$ is the reflecting direction, $n$ is the specular reflection exponent and typically varies from 1 to several hundred, depending on the surface material. Referring to Eq. 3, the lighting intensities $I_{a}$ and $I_{p o}$, and the lighting direction $\vec{L}$ are constant. The variables are the albedos $k_{a}, k_{d}, k_{s}, n$, and the surface norm $\vec{N}$. The albedos and surface norms are different at different surface points. $n$ is assumed to be constant [4] and is set to 2 [9]. The norm of a given point on the face surface is calculated using 3D shape models generated in Section 2.

It can be observed from Eq. 3 that the albedos and the lighting intensities cannot be separated only knowing pixel values of images. Since lighting intensities $I_{a}$ and $I_{p o}$ are constant across the face surface, we redefine the reflection components as

$$
H_{a}=I_{a} k_{a}, \quad H_{d}=I_{p o} k_{d}, \quad H_{s}=I_{p o} k_{s} .
$$

Given frontal and profile views under a parallel frontal lighting $\vec{L}$, we have $\vec{L}=\vec{V}_{F}$. To estimate the three reflection components, the frontal face view is used and for point $i$ Eq. 3 becomes

$$
I_{F, i}=H_{a, i}+H_{d, i}\left(\vec{V}_{F} \cdot \vec{N}_{i}\right)+H_{s, i}\left(\vec{R}_{i} \cdot \vec{V}_{F}\right)^{2},
$$

where $\vec{V}_{F}$ is the frontal direction.
We first assume points have similar reflection properties within the facial area and set the reflection components uniform for all the pixels as $H_{a}=H_{a, i}, H_{d}=H_{d, i}$, and $H_{s}=H_{s, i}$ for all $i$. Therefore,

$$
\left\{\begin{array}{l}
I_{F, 1}=H_{a}+H_{d}\left(\vec{V}_{F} \cdot \vec{N}_{1}\right)+H_{s}\left(\vec{R}_{1} \cdot \vec{V}_{F}\right)^{2} \\
I_{F, 2}=H_{a}+H_{d}\left(\vec{V}_{F} \cdot \vec{N}_{2}\right)+H_{s}\left(\vec{R}_{2} \cdot \vec{V}_{F}\right)^{2} \\
\vdots \\
I_{F, m}=H_{a}+H_{d}\left(\vec{V}_{F} \cdot \vec{N}_{m}\right)+H_{s}\left(\vec{R}_{m} \cdot \vec{V}_{F}\right)^{2},
\end{array}\right.
$$

where $m$ is the pixel count. Eq. 6 is solved using the Least Square method and three reflection components $H_{a}, H_{d}$, and $H_{s}$ are obtained. Since face surfaces have spatially variable reflection properties, the following refinement process is then applied to calculate actual reflection components for each pixel. An estimated frontal view $I_{F}^{\prime}$ is first generated using the calculated reflection components $H_{a}, H_{d}$, and $H_{s}$. A refinement ratio $c_{i}$ is defined as $c_{i}=I_{F, i} / I_{F, i}^{\prime}$ which is the difference between the estimated pixel value and the actual value of the gallery image. Then, the actual reflection components are computed as $H_{a, i}^{*}=c_{i} H_{a}, H_{d, i}^{*}=c_{i} H_{d}$, and $H_{s, i}^{*}=c_{i} H_{s}$.

After the texture analysis, textures of any virtual view $I_{\theta}$ under arbitrary viewing direction $\theta$ can be generated based on Phong reflectance model as

$$
I_{\theta, j}=H_{a, j}^{*}+H_{d, j}^{*}\left(\vec{L}_{\theta} \cdot \vec{N}_{j}\right)+H_{s, j}^{*}\left(\vec{R}_{j} \cdot \vec{V}_{\theta}\right)^{2},
$$

where $\theta$ is the yaw rotation from the frontal direction, $I_{\theta, j}$ is the pixel value of $j$ th point on the virtual view.

\section{Experiment and results}

In this paper, the proposed virtual view synthesis approach is combined with a view-based principle component analysis (PCA) algorithm to form a complete poseinvariant face recognition system. In experiment, the proposed system is tested on 30 images taken from the CMUPIE database [11]. For each individual, two orthogonal face images, i.e., frontal and profile views, are used as gallery views. Using the face shape modeling algorithm in Section 2 and texture synthesis algorithm in Section 3, arbitrary views from different viewpoints were synthesized. A comparison between the real probe view of a face and a virtual view generated by our proposed algorithm is shown in Fig. 3.

Then we applied face recognition on the synthesized virtual views with real face views under different poses. The resolution of both the virtual views and the rotated real views is $160 \times 160$ pixels. Fig. 4 shows the frontal real view, the generated virtual view and the real face view of a person. The real face view under $15^{\circ}$ rotation was taken in the same lighting condition with the frontal view. The background light remained unchanged and the flash light is from 


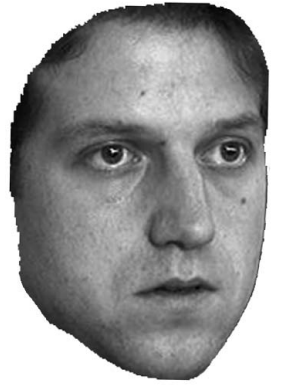

(a)

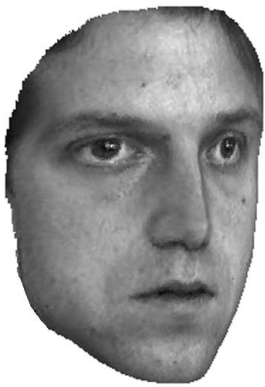

(b)
Figure 3. The comparison between the virtual view (b) synthesized using the proposed approach and the corresponding real view (a).

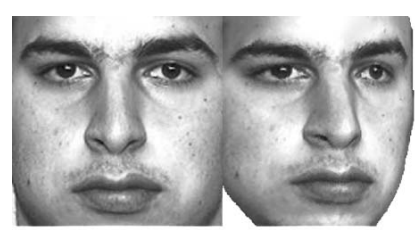

(a)

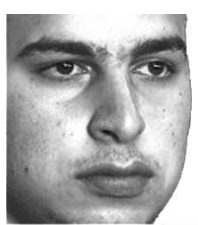

(c)
Figure 4. Frontal (a) and rotated (c) real views and a synthesized virtual view (b) for viewbased face recognition.

the frontal direction of the face. The synthesized virtual views (Fig. 4b) were used as gallery views and rotated real views (Fig. 4c) as probe views. The algorithm compared the gallery views with the probe views. Then the rotated probe views (Fig. 4c) were matched with the frontal views (Fig. 4a) directly. The recognition results are summarized in Table 1. It shows that small pose variations $\left(15^{\circ}\right.$ in this experiment) affect face recognition performance dramatically. And the proposed pose-invariant face recognition improves the recognition rate against pose variations.

Table 1. Face recognition performance

\begin{tabular}{|c|l}
\hline frontal view $v s$. probe view & virtual view $v s$. probe view
\end{tabular}
$46.67 \%$ $100.00 \%$

\section{Conclusion}

In this paper, we have presented a pose-invariant face recognition approach based on virtual views generated from face shape modeling and texture analysis using two orthogonal gallery images, i.e., frontal and profile views. In shape modeling step, a feature-based multilevel quadratic variation minimization approach is applied to generate a 3D personalized face shape. In texture analysis step, facial reflectance components are estimated based on Phong reflectance model from 2D images and used to synthesize virtual textures under novel poses. By considering specular reflection, the accuracy of texture synthesis is improved compared to conventional Lambertian reflectance model. Virtual face views are then synthesized and a PCA face recognizer is applied using the virtual face views as gallery to recognize rotated faces. The experimental results show that the proposed method provides a solution to the problems of recognizing faces under differed poses. The future work is to perform view-based face recognition under more viewing directions and lighting conditions.

\section{References}

[1] Face recognition vendor test. http://www.frvt.com/.

[2] D. Beymer and T. Poggio. Face recognition from one example view. ICCV, pages 500-507, 1995.

[3] V. Blanz and T. Vetter. Face recognition based on fitting a 3D morphable model. PAMI, 25(9):1063-1074, Sept. 2003.

[4] T. Cootes, G. Wheeler, K. Walker, and C. Taylor. Viewbased active appearance models. Image and Vis. Comput., 20(9-10):657-664, Aug. 2002

[5] P. Debevec, T. Hawkins, C. Tchou, H.-P. Duiker, W. Sarokin, and M. Sagar. Acquiring the reflectance field of a human face. SIGGRAPH, pages 145-156, 2000.

[6] A. S. Georghiades, P. N. Belhumeur, and D. J. Kriegman. From few to many: illumination cone models for face recognition under variable lighting and pose. PAMI, 23(6):643660, June 2001

[7] W. E. L. Grimson. An implementation of a computational theory of visual surface interpolation. CVGIP, 22(1):39-69, Apr. 1983.

[8] R. Gross, I. Matthews, and S. Baker. Appearance-based face recognition and light-fields. PAMI, 26(4):449-465, Apr. 2004.

[9] H. H. S. Ip and L. Yin. Constructing a 3D individualized head model from two orthogonal views. Vis. Comput., 12(5):254-266, 1996.

[10] B. T. Phong. Illumination for computer-generated pictures. Comm. ACM, 18(6):311-317, June 1975.

[11] T. Sim, S. Baker, and M. Bsat. The CMU pose, illumination, and expression database. PAMI, 25(12):1615-1618, Dec. 2003

[12] X. Zhang, Y. Gao, and M. K. H. Leung. Multilevel quadratic variation minimization for $3 \mathrm{D}$ face modeling and virtual view synthesis. Proc. Multimedia Modeling, 1:132-138, 2005

[13] W. Zhao, R. Chellappa, P. Phillips, and A. Rosenfeld. Face recognition: a literature survey. ACM Comput. Surveys, 35(4):399-459, Dec. 2003. 